\title{
Le programme aéronautique Skylander en Lorraine : des intérêts d'acteurs convergents
}

The aerospace program Skylander in Lorraine: convergent interests for stakeholders

Das Luftfahrtprojekt Skylander in Lothringen : übereinstimmende Akteurinteresse

\section{Colette Renard-Grandmontagne}

\section{(2) OpenEdition}

\section{Journals}

Édition électronique

URL : http://journals.openedition.org/rge/3001

DOI : $10.4000 /$ rge.3001

ISSN : 2108-6478

\section{Éditeur}

Association des géographes de l'Est

Édition imprimée

Date de publication : 31 décembre 2010

ISSN : 0035-3213

\section{Référence électronique}

Colette Renard-Grandmontagne, «Le programme aéronautique Skylander en Lorraine : des intérêts d'acteurs convergents », Revue Géographique de l'Est [En ligne], vol. 50 / 3-4 | 2010, mis en ligne le 09 octobre 2011, consulté le 08 septembre 2020. URL : http://journals.openedition.org/rge/3001 ; DOI : https://doi.org/10.4000/rge.3001

Ce document a été généré automatiquement le 8 septembre 2020

Tous droits réservés 


\section{Le programme aéronautique Skylander en Lorraine : des intérêts d'acteurs convergents}

The aerospace program Skylander in Lorraine: convergent interests for

stakeholders

Das Luftfahrtprojekt Skylander in Lothringen : übereinstimmende

Akteurinteresse

Colette Renard-Grandmontagne

\section{Introduction}

1 Le 4 septembre 2008, le PDG de GECI International, Serge Bitboul, a annoncé officiellement l'implantation d'une de ses filiales, Sky Aircraft, à Chambley-Bussières pour y développer un projet industriel de haute technologie, à savoir la conception puis la fabrication d'un nouvel avion baptisé Skylander. L'information a pu surprendre, à plus d'un titre, journalistes et géographes notamment parce que la Lorraine n'a pas une tradition industrielle aéronautique ${ }^{1}$. Antérieurement à cette annonce, d'autres lieux d'implantation du projet avaient été pressentis. Nantes, la Roumanie où GECI a un bureau d'étude et où fut, semble-t-il, esquissé le premier dessin de l'avion ont été cités par la presse ${ }^{2}$. Plus durablement, et successivement, ont été étudiés les dossiers de Tarbes, puis d'Evora au Portugal de 2004 à 2008 et enfin celui de Chambley Air Base, ancienne base américaine de l'OTAN aménagée dans la campagne lorraine où le projet a rapidement pris corps. La multiplicité des points de chute énumérés pourrait laisser à penser qu'une telle implantation peut être réalisée n'importe où en France ou en Europe et que l'entreprise, complètement émancipée des contraintes d'un espace aboli, peut choisir sans obstacle sa localisation (Fache, 2002). Pourtant, officiellement un des acteurs principaux du projet, la Région Lorraine, affirme au contraire que «l'arrivée de Sky Aircraft à Chambley ne doit rien au hasard $»^{3}$. Rechercher les conditions rendant 
possibles le choix d'une localisation et celles qui ont réellement joué un rôle lors du choix (c'est-à-dire les facteurs) a été longtemps l'objet premier des travaux des géographes (Mérenne-Schoumaker, 2002). La relation entre acteurs économiques et politiques, entre entreprises et territoires, est au cœur des réflexions en géographie économique depuis près de trente ans (Fischer A., 1999, Bonnet et Broggio, 2010).

2 Cette contribution tentera de décrypter le jeu des acteurs économiques et politiques qui a finalement poussé la société GECIà arrêter sa stratégie géographique, c'est-à-dire à choisir le territoire lorrain. A cette fin, a été analysée l'abondante revue de presse constituée par le service Documentation du conseil régional de Lorraine et de longs entretiens ont été conduits avec les principaux acteurs économiques et institutionnels de ce programme. Après une rapide présentation de la société et du programme Skylander, nous essaierons de dégager les intérêts partagés de GECI et de la Région Lorraine et leurs engagements respectifs. Ce projet qui se différencie nettement des autres implantations industrielles que la Lorraine a accueillies au cours des trente dernières années, dans un contexte quasi-permanent de «conversion-diversificationrestructuration " Fischer, 1999), constitue un pari industriel qui doit, pour réussir, affronter et surmonter plusieurs défis.

\section{GECI Aviation, Sky Aircraft, Skylander : en passant par Chambley Planet'Air}

\section{A. Une entreprise innovante}

3 Entreprise de haute technologie, GECI International, dont le siège social est établi à Paris, a été fondée par Serge Bitboul en 1980. La société dont le capital est détenu directement et indirectement par son fondateur, à hauteur de $42 \%$, est cotée sur le marché boursier Euronext depuis 2001.Le tableau suivant rend compte de la répartition du capital et des droits de vote, à la clôture de l'exercice annuel 2009-2010 (tableau 1).

Tableau 1 : Répartition du capital et des droits de vote de Geci International, 31 mars 2010

\begin{tabular}{|l|c|c|}
\hline & \% du capital & \% des droits de vote \\
\hline Serge Bitboul & 16,3 & 20,3 \\
\hline GTH* $^{*}$ & 25,80 & 36,2 \\
\hline Auto-détention & 0,3 & 0,3 \\
\hline Autres nominatifs & 10,1 & 7,8 \\
\hline Autres au porteur (actionnariat flottant) & 47,4 & 35,4 \\
\hline
\end{tabular}

GTH* GECI Technologies Holding SAS : société contrôlée par S. Bitboul

Source : d'après Geci International, Rapport financier annuel exercice 2009-2010

4 Cette PME de près de 900 salariés, dont $85 \%$ d'ingénieurs et cadres ${ }^{4}$,exerce ses activités de conseil et de développement en ingénierie, principalement dans les domaines aéronautique et naval mais aussi dans les transports terrestres, l'énergie et le génie civil. Labellisée depuis novembre 2006 "entreprise innovante" par OSEO/ANVAR, l'entreprise travaille pour les grands donneurs d'ordre que sont EADS, Dassault, SNECMA, British Aerospace. Pour ce faire, elle dispose d'une vingtaine de bureaux 
essentiellement implantés dans des métropoles, celles des grands foyers aéronautiques européens (Toulouse, Bordeaux, région parisienne, Munich, Brême, Hambourg, Londres, Madrid) ${ }^{5}$. La France et l'Allemagne sont les deux principaux espaces de travail de la société (figure 1).

Figure 1 : Répartition du chiffre d'affaires de GECI International en 2009-2010

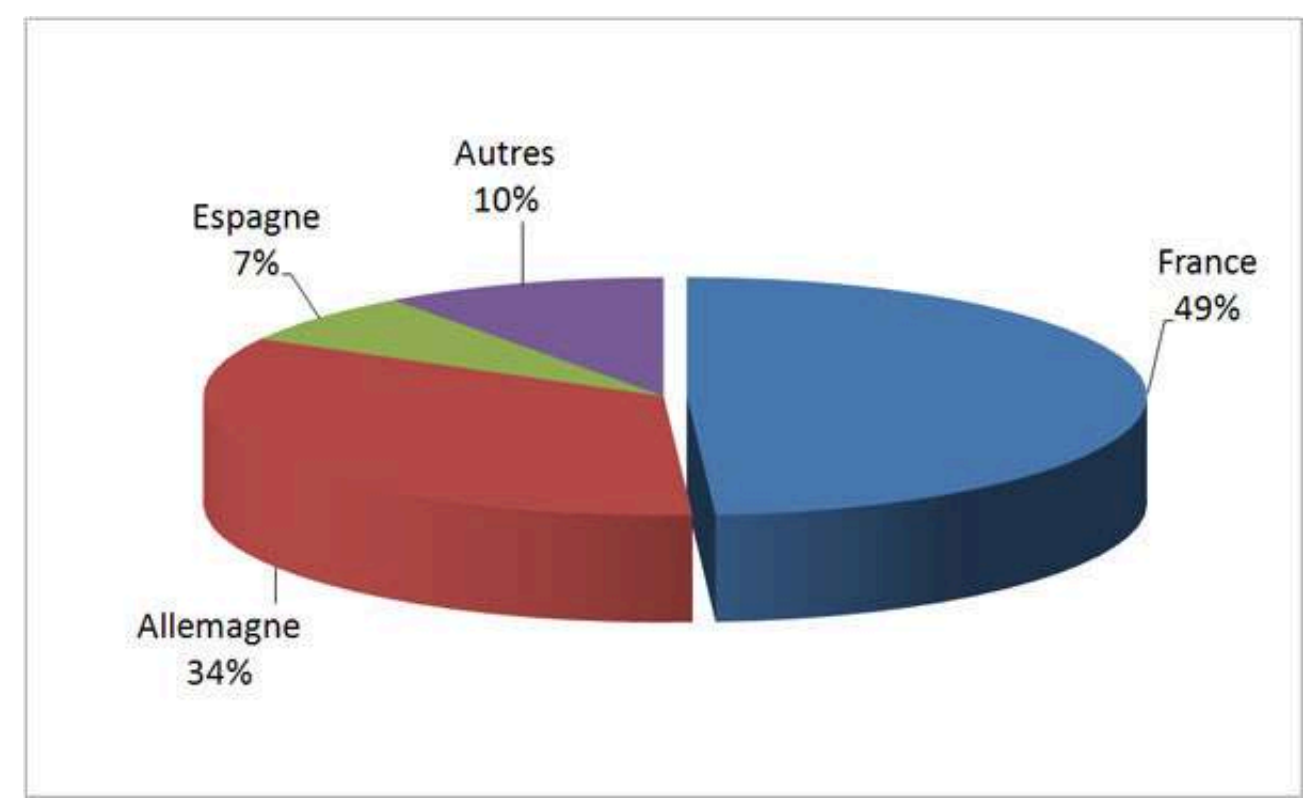

Source : Geci International

5 Après vingt ans d'investissement en recherche et développement aéronautique, GECI a décidé de capitaliser ses savoir-faire en produisant ses propres avions. L'entreprise du « futur en mouvement » comprend désormais deux pôles (figure 2) : le pôle traditionnel GECI Ingénierie et le nouveau pôle GECI Aviation créé en mars 2010 (filiale détenue à 88,6 \% par GECI International). Ce second pôle est lui-même composé de deux filiales :

6 - Sky Aircraft, née en novembre 2008, pour concevoir puis mettre en œuvre le programme Skylander SK 105,

7 - Reims Aviation Industries, constructeur d'avions depuis 1933 qui, associé à la société américaine Cessna, a assemblé en France les avions légers monomoteurs (Cessna 152 et Cessna 172) de 1962 à 1989 ; il produit aussi depuis le milieu des années 1980, pour son propre compte, l'avion F 406, appareil destiné à la surveillance aérienne des trafics illégaux (drogue, contrebande, piraterie), de l'immigration clandestine, à celle des zones de pêche et à la détection des diverses pollutions marines. GECI est devenu actionnaire majoritaire de Reims Aviation, en 2008, en prenant le contrôle de 59\% du capital de la société champenoise (environ 70 salariés). Depuis le 26 mars 2010, Reims Aviation Industries est une filiale dont le capital est détenu à 100\% par GECI Aviation. 
Figure 2 : Organigramme simplifié de GECI International

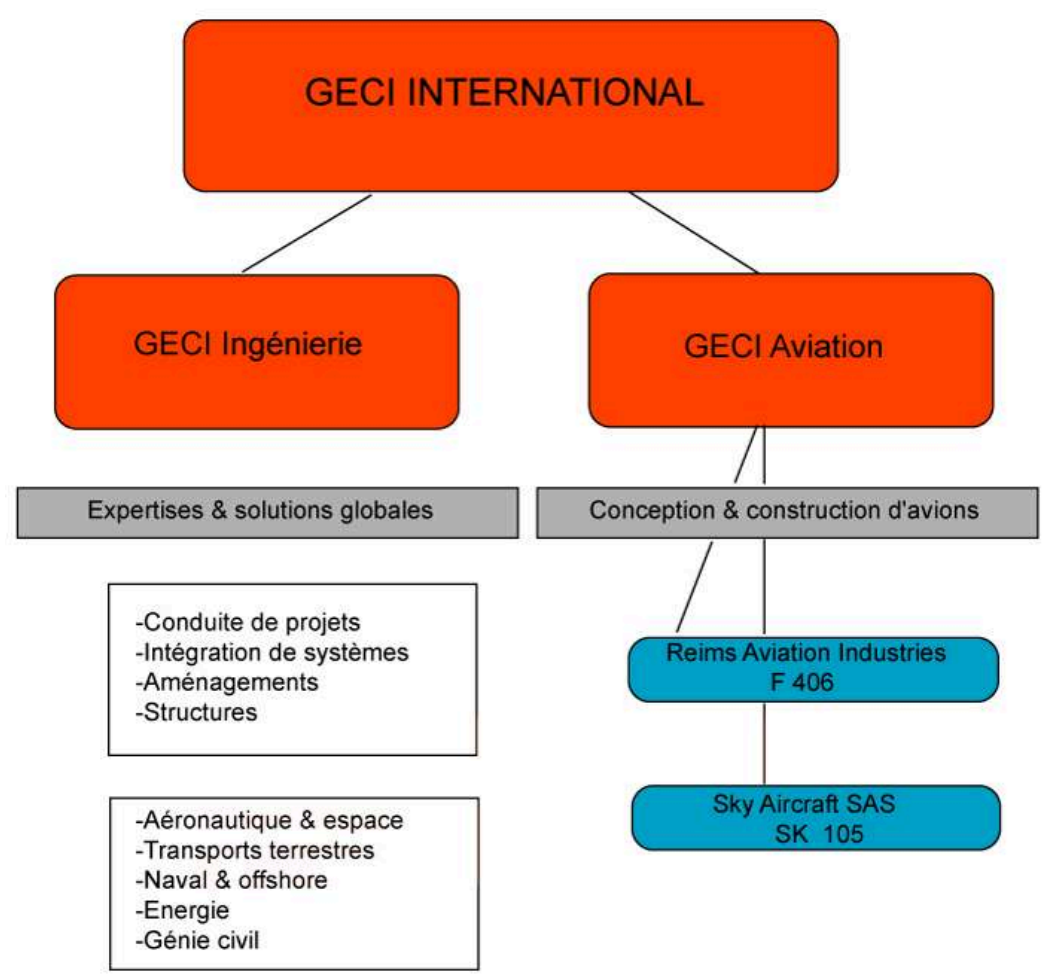

8 Le chiffre d'affaires du pôle Aviation pour l'exercice annuel 2009-2010 fut de 7,7 millions d'euros, soit $13 \%$ du chiffre d'affaires total de GECI International (58,6 millions $€)$. L'objectif est d'atteindre, à court terme, la répartition suivante : $20 \%$ pour GECI Aviation, $80 \%$ pour le pôle ingénierie.

9 Par ces deux productions, effective pour l'une et à venir pour l'autre, GECI est entré dans le club assez fermé des avionneurs.

\section{B. Le programme Skylander}

10 Depuis novembre 2008, une équipe de 40 personnes élargie à 180 salariés (fin 2010) travaille à la conception du Skylander SK 105 (photo 1), avion tout terrain de nouvelle génération, rustique et polyvalent destiné aux marchés du transport à courte distance, $\mathrm{du}$ fret et de l'humanitaire. Cet avion bi-turbopropulseurs léger, surnommé « le Logan des airs ", est un avion utilitaire pouvant transporter 19 passagers ou 3 tonnes de fret. Le prototype est attendu pour fin 2011-début 2012. La livraison des premiers avions devrait avoir lieu au cours de l'année 2015 et la prévision est d'assembler 9 avions par mois, à Chambley, à partir de 2016. A terme, Sky Aircraft devrait créer 310 emplois directs et 700 emplois indirects.

11 Ce projet est innovant pour GECI qui découvre pour la première fois les arcanes de la conception d'un avion dans sa totalité ; il l'est pour la Lorraine aussi qui a accueilli davantage d'établissements d'assemblage ou de montage, qualifiés parfois "d'usines tournevis », dans l'industrie automobile ou l'électronique. 
Photo $1:$ Un avion rustique polyvalent

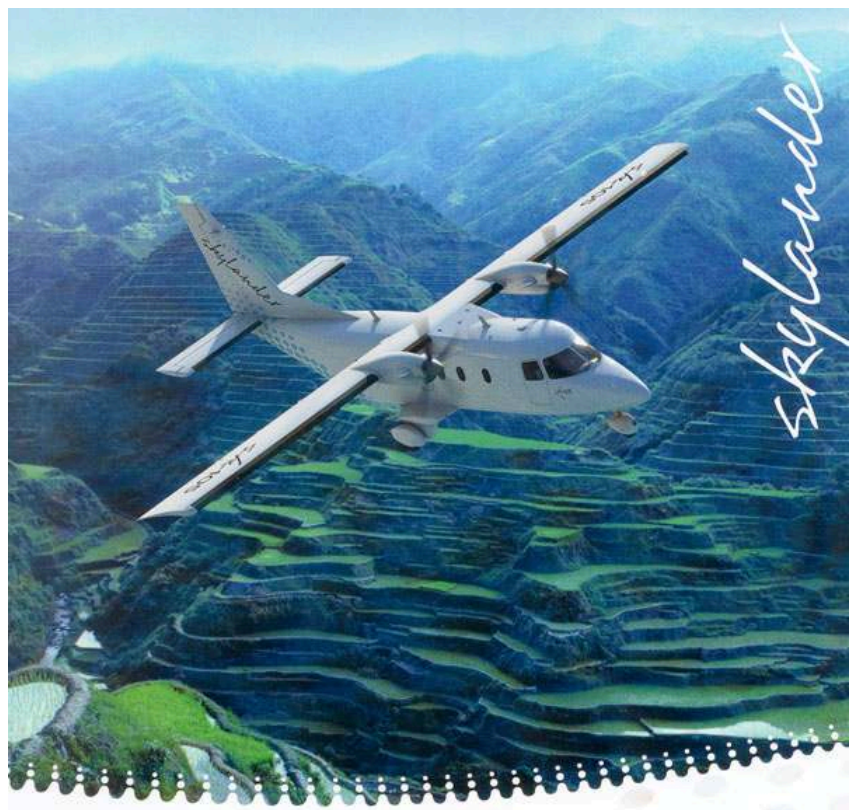

Skylander SK-105

Next generation

twin-turboprop transporter

Cet avion prévu pour transporter 19 passagers ou 3 tonnes de fret est destiné aux marchés du transport à courte distance (1500 km)

(c) GECI International

12 Comment expliquer qu'une entreprise jusqu'alors implantée dans des métropoles où elle a développé des réseaux relationnels en termes d'activité aéronautique ait finalement opté pour la Lorraine et plus encore pour un environnement rural ?

\section{Intérêts partagés de GECI Aviation et de la Région Lorraine : la rencontre de deux projets}

La communication officielle qui, pendant plus de dix-huit mois, a alimenté les articles de la presse écrite régionale et dans une moindre mesure nationale voire internationale est en fait une partition à deux voix. Elle se compose d'une part des communiqués de presse de l'entreprise qui, dans un système économique libéral, est l'acteur majeur prenant l'initiative et ayant le pouvoir de décision d'une implantation, et d'autre part de la communication de la Région Lorraine qui apparaît d'emblée comme un acteur essentiel du projet : communication constituée de déclarations de son président, JeanPierre Masseret (PS), de présentations du projet en séances plénières du conseil et de plaquettes d'information sur Chambley Planet'Air.

Les deux discours officiels convergent pour mettre en valeur une collectivité régionale entreprenante, qui «a su se montrer attractive pour accueillir ce projet innovant » et qui a proposé, conjointement avec l'Etat français, « une opportunité » à une entreprise en cours d'implantation au Portugal. Nous ne sommes pas dans une configuration courante dans laquelle l'entreprise prospecte et met en concurrence plusieurs 
territoires susceptibles de lui convenir, comme ce fut le cas pour MCC, en 1997, (usine de la Smart, Renard-Grandmontagne, 2004) ou Toyota (usine d'Onnaing, près de Valenciennes), mais dans celle d'une entreprise qui s'est laissée séduire par l'offre d'une collectivité pour venir s'installer dans son territoire.

\section{A. Quels éléments d'attraction, la Région met-elle en avant ?}

15 La Région présente comme "un atout déterminant » un savoir-faire industriel sous la forme "d'un potentiel de sous traitants de toutes les filières industrielles ». La mise en lumière d'un savoir-faire n'est pas étonnant dans une région anciennement industrialisée (R.A.I., Fischer, 1999), à tradition forte dans le travail des métaux. En revanche, la mise sur le devant de la scène d'un cluster aéronautique, créé en 2005 et baptisé Aériades peut, à priori, l'apparaitre davantage. Cette grappe d'entreprises, membre du GIFAS (Groupement des Industries Françaises Aéronautiques et Spatiales) regroupe une trentaine de partenaires (2 500 salariés environ) dont une partie plus ou moins importante de l'activité correspond à de la sous-traitance de deuxième rang pour les filières aéronautique et navale (PME d'électronique, de plasturgie, d'optique laser, de métrologie et des écoles supérieures telles l'Ensam et Supélec) ${ }^{6}$. L'identification et la fédération des industriels lorrains dans le domaine de la production aéronautique ont été amorcées au début des années 2000, à l'initiative de l'association ARES (Armée, Recherche, Economie et Sciences) crééeen Franche Comté en 1989 et dont une délégation fut ouverte en 1997, à Lunéville, vieille cité militaire lorraine. Partant du constat que la Lorraine industrielle recèle des compétences de très haut niveau dont elle ne tire pas suffisamment parti, il s'agissait de promouvoir les capacités technologiques des PME-PMI régionales sous-traitantes de la Défense Nationale et de favoriser les transferts de technologie entre ces entreprises et les grands groupes d'Etat (Aérospatiale, Airbus,...). Le projet de l'ARES, accepté par le conseil régional de Lorraine en novembre 2000, visait à créer un pôle aéronautique et spatial sur l'emprise même de l'aéroport régional de Lorraine où plusieurs implantations et innovations étaient prévues (Matra, fabrication d'un micro hélicoptère).

16 Aériades, cluster sans grosse locomotive à la différence d'autres clusters régionaux ou du pôle de compétitivité Matéralia (ex-MIPI), répond comme ces derniers à une volonté de modifier l'image de la région en matière de production industrielle. Il s'agit de substituer à la représentation de vieilles productions industrielles en déclin telles celles de la sidérurgie, qui peut néanmoins être innovante et performante, celle du développement d'une nouvelle filière de haute technologie.

17 La deuxième série de facteurs attractifs est relative au site d'implantation au sein de l'espace régional (échelle micro-spatiale). Il va de soi qu'une entreprise construisant un nouvel avion recherche la proximité d'un aérodrome fonctionnel, mais peu fréquenté et dont l'activité aérienne n'entrera pas en conflit avec la circulation commerciale de grands aéroports. La Lorraine est dans ce domaine fort bien dotée du fait de son équipement d'aérodromes militaires hérités pour la plupart des anciennes bases aériennes de l'OTAN, soit totalement désertées dès la fin des années 1960, soit reprises plus ou moins durablement par l'armée française (Renard-Grandmontagne, 2010). Cinq ou six sites aéroportuaires lorrains étaient susceptibles de convenir en intégrant aussi l'aérodrome militaire de la base aérienne 128 de Metz-Frescaty fermée en 2011 et l'aéroport civil Metz-Nancy-Lorraine, à quelques encablures de la gare TGV Lorraine. La 
désignation du site d'élection, Chambley, est un acte politique lorrain de gouvernance territoriale qui s'inscrit dans le schéma régional de développement économique adopté en juin 2006 dont les idées maîtresses sont (figure 3) :

- l'estompage du clivage Lorraine du Nord/Lorraine du Sud,

- la structuration de l'espace régional par le sillon mosellan dominée par les deux métropoles Nancy et Metz

- mais aussi le soutien aux « territoires en devenir », en particulier le long d'un axe est-ouest allant de la métropole Sarrebrück-Moselle Est (ex bassin houiller) au lac de La Madine, en passant par l'Espace Central métropolitain (Conseil Régional, 2006). Cette vaste zone dite « Espace Central » se situe « au centre des réseaux de transports qui contribuent à l'irrigation solidaire de la Lorraine ».Considéré comme espace à « enjeu stratégique », « l'aménagement de l'Espace Central métropolitain consiste en un développement concerté confortant le rôle majeur du Sillon Lorrain par l'installation d'un lieu de vie et d'activités, relais complémentaire et différencié de l'offre des agglomérations contribuant à une lisibilité accrue de la Lorraine dans l'espace européen 》. Chambley appartient à cet Espace Central.

- une« métropole lorraine équilibrée dans ses territoires » sous entendu urbains et ruraux.

Figure 3 : Chambley Planet'Air au cœur de l'Espace Central lorrain

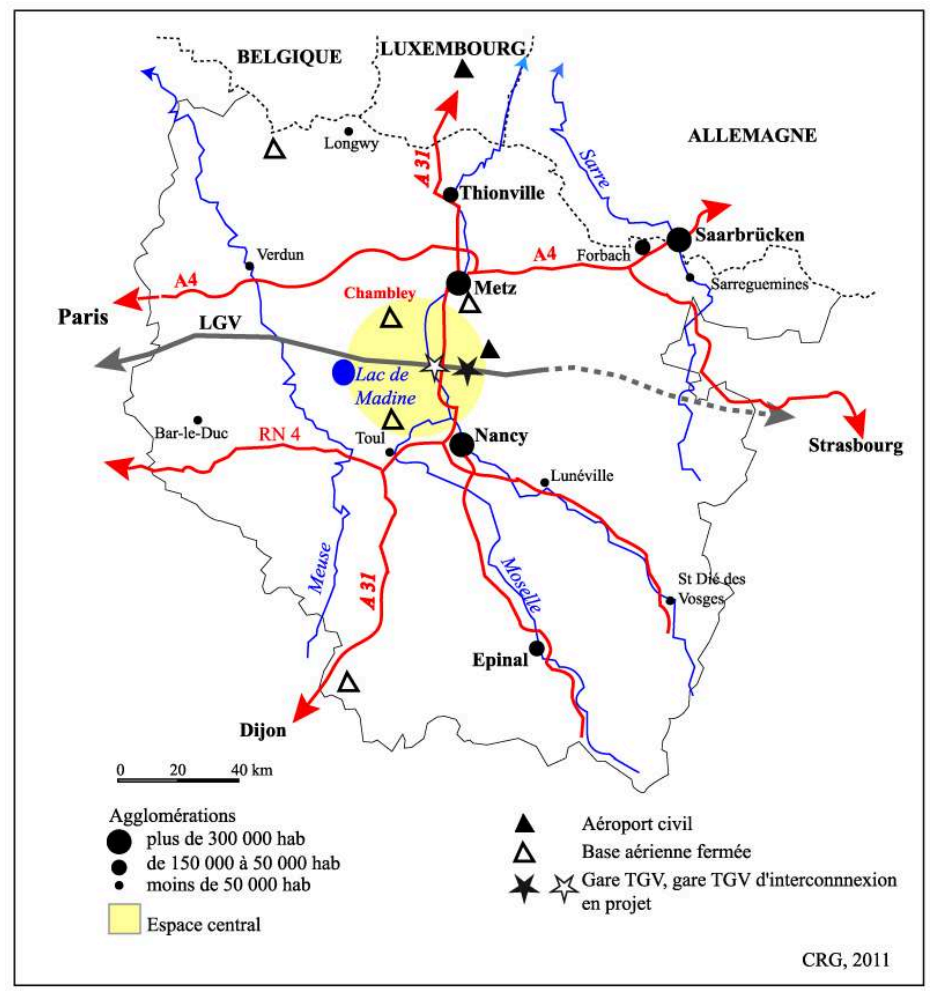

L'implantation de Sky Aircraft à Chambley s'inscrit donc alors dans un projet de développement local, en devient le fer de lance et stimule sa réalisation. Depuis le début des années 2000, la Région Lorraine a entrepris les démarches nécessaires pour acquérir la friche militaire aérienne de Chambley (486 ha) afin d'y réaliser ce qu'elle a alors pompeusement nommé « la première ville aéronautique au monde ». Il s'agit d'un grand projet aéronautique à la fois de production et de loisirs qui a imposé nécessairement une phase de requalification. Le nouvel espace requalifié est partagé, 
de part et d'autre de la piste, entre différents usagers et différentes fonctions (figure 4, photo 2):

- au nord de la piste, un espace dédié à des activités permanentes de loisirs aéronautiques (pratique d'ULM, de planeurs, d'hélicoptères et d'aviation légère) et à des manifestations événementielles tel Lorraine Mondial Air Ballons qui rassemble, depuis 1989, tous les deux ans, des montgolfières venues de très nombreux pays.

- au sud de la piste, un espace subdivisé en deux zones d'activités économiques, intégrant quelques établissements déjà installés ${ }^{7} e t$ devant accueillir de nouvelles activités.

19 La Région possède donc là un foncier disponible dont 22 ha ont été mis à la disposition de GECI.

Figure 4 : Chambley-Planet'Air : un espace partagé

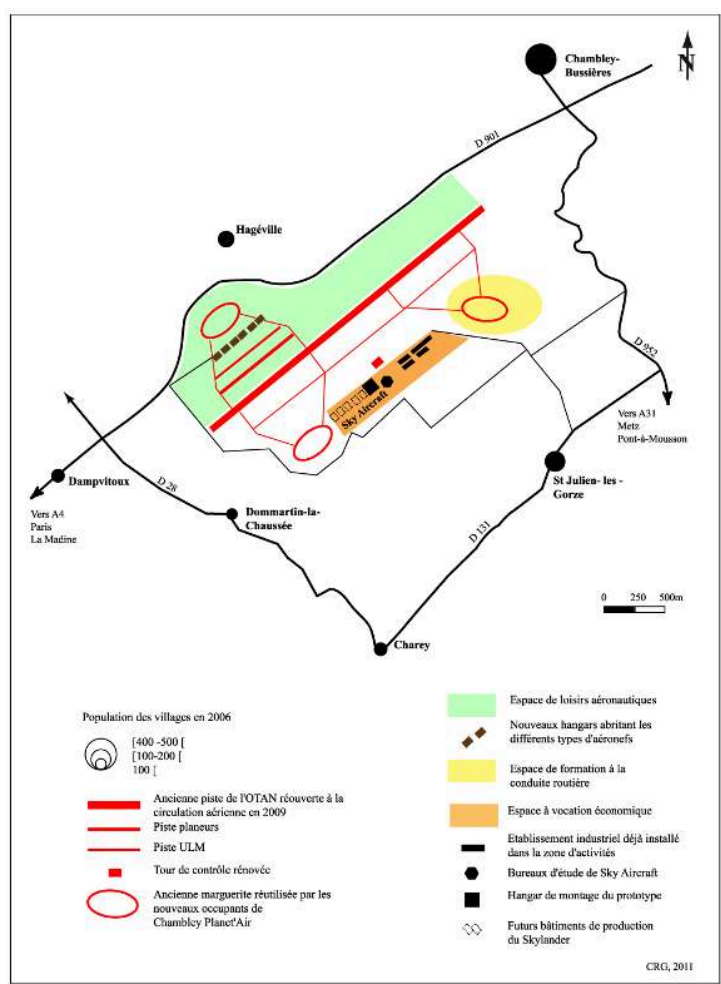


Photo 2 : Chambley Planet'Air

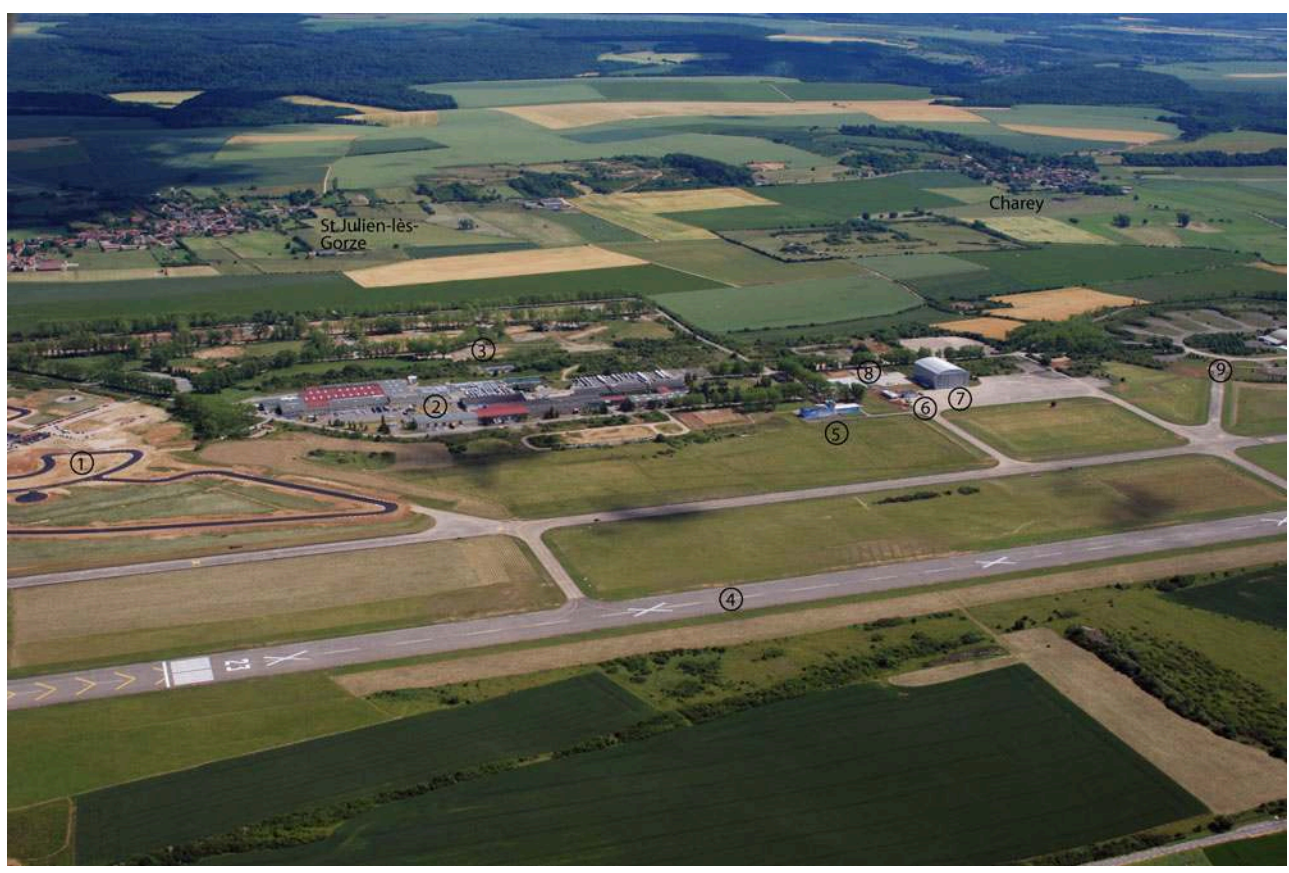

Dans un environnement rural de faible densité, l'ancienne friche de Chambley Air Base a été requalifiée. Au-delà de la piste réouverte à la circulation aérienne, en 2009, sont aménagées les zones d'activités économiques ; elles sont déjà en partie occupées notamment par Sky Aircraft dont les nouveaux bâtiments sont prévus entre le hangar et l'ancienne marguerite : 1-Espace de formation à la conduite, 2-Menuiserie industrielle Azur Production, 3-Ancienne zone de vie requalifiée, 4-Piste orientée NE/SW, 5-Tour de contrôle rénovée, 6- Maison de la Prospective, 7- Hangar d'assemblage des prototypes, 8- Bureaux provisoires de Sky Aircraft, 9-Ancienne marguerite réutilisée par Sky Aircraft

Cliché : A. Humbert et C. Renard-Grandmontagne, 2009

\section{B. Les « opportunités » décisives}

L'apport foncier fait sans aucun doute partie des opportunités offertes par la Région. Ce n'est évidemment pas la plus importante. La conduite d'un projet de conception et de construction d'un avion même simple nécessite un investissement considérable (coût total du projet estimé à 130 millions d'euros en 2008) qu'une entreprise junior dans le cercle des avionneurs peine à rassembler. Les aides publiques, sous toutes leurs formes, apparaissent indispensables à la réalisation du projet. Les pérégrinations du projet Skylander nous semblent en fait étroitement liées à l'obtention d'aides publiques «territorialisées » et à leur délai d'attribution. Si le montant de l'aide publique est encadré en fonction du zonage (territoire classé zone AFR, Aide à Finalité Régionale) ${ }^{8}$, la rapidité d'avancement des dossiers de demande de subventions est plus variable, dépendant entre autre de la détermination de l'engagement des acteurs institutionnels, de la mobilisation de leurs réseaux politiques et du contexte temporel d'attribution. La très grande réactivité de la Région Lorraine et de l'Etat français (quelques mois voire quelques semaines) apportant respectivement 2 millions et 2,5 millions d'euros l'ont emporté sur les lenteurs lusitaniennes où le projet Skylander avait pourtant été classé «projet industriel national» et où il aurait pu bénéficier d'aides européennes supérieures.

21 Comment expliquer, qu'en quelques mois (printemps - automne 2008), les contacts établis entre Geci et la Région Lorraine par la CEIS (Compagnie Européenne 
d'Intelligence Scientifique), véritable cheville ouvrière, aient été transformés en acte officialisé? Cette période coïncide avec celle de l'annonce des projets de restructuration militaire et de redéploiement des forces sur le territoire national, prévus par la Loi de Programmation Militaire (2009-2014). A ce titre, la Lorraine est la région française la plus affectée : 7000 emplois supprimés ou transférés vers des latitudes plus méridionales (Toulouse ou Montpellier par exemple), 700 emplois créés en contrepartie. Après avoir subi les traumatismes successifs des fermetures industrielles (textiles, minières, sidérurgiques), la région doit affronter à nouveau, par choix de l'Etat, l'anémie de certains de ses territoires (Dieuze, Commercy par exemple). L'attribution d'une prime à l'aménagement du territoire par l'Etat peut être considérée comme une aide à une nouvelle reconversion'. De manière tout à fait anecdotique, l'implantation du projet Skylander en Lorraine a été officialisée le surlendemain de la réception, par le Président de la République, d'une délégation d'élus mosellans très inquiets du devenir de leurs territoires ${ }^{10}$. Cette annonce de circonstance mais préparée durant les mois précédents indique que d'autres facteurs que ceux mis en avant dans la communication officielle sont intervenus dans l'implantation de Sky Aircraft. Si la reconstitution de la séquence chronologique ne permet guère d'en douter, la relation entre le déménagement des régiments et l'implantation de GECI reste néanmoins difficile à établir de façon certaine.

Avec l'appui de la Région et l'intervention probablement décisive de l'Etat par l'attribution d'une prime à l'aménagement du territoire, le projet a pris forme, dès novembre 2008, dans des locaux provisoires construits par la Région et loués à Sky Aircraft. L'aventure industrielle est née de la rencontre d'intérêts partagés: la recherche d'une entreprise emblématique dans une filière innovante pour l'un, la recherche de partenaires publics prêts à accompagner un projet industriel risqué pour les autres. Si l'environnement économique (proximité de sous-traitants) a pu influer, les interventions des pouvoirs publics (interventions des responsables régionaux, interventions régionalisées du pouvoir central) furent décisives. En revanche, le facteur cadre de vie (conditions de logement, présence d'équipements en particulier culturels et récréatifs, facilité d'accès à la métropole lorraine) a probablement été sous estimé.

\section{Un pari industriel à réussir}

La réalisation du Skylander est une aventure passionnante pour ceux et celles qui y participent, mais c'est aussi une construction difficile. Parmi les difficultés voire les défis que l'entreprise a à surmonter, trois principaux peuvent être retenus.

\section{A. Rassembler de nouveaux capitaux}

24 La difficulté récurrente, qui apparaît comme un défi permanent, est la recherche de capitauxpour financer les investissements nécessaires à la réalisation du programme. Ce problème du financement n'est pas spécifique à GECI mais est partagé par le plus grand nombre de petites et moyennes entreprises en France depuis plus de cinquante ans (Bonnet et Broggio, 2010).La quête est rendue peut être encore plus aiguë par le contexte de crise économique et par la frilosité des banques à prêter aux entreprises de taille moyenne. De ce fait, l'acteur politique et institutionnel est sollicité à nouveau pour accompagner davantage l'entreprise dans le développement du programme. 
L'accompagnement de la Région s'est amplifié depuis la réélection de son président, au printemps 2010. L'aide est tout d'abord financière. La Région a consenti à Sky Aircraft une avance de 9,1 millions d'euros remboursable en cinq ans qui a été substituée à la subvention de 2 millions d'euros accordée fin 2008. Le Président de Région a également obtenu de l'assemblée l'autorisation d'une demande au Conseil d'Etat permettant l'entrée de la Région Lorraine dans le capital de GECI Aviation, à hauteur de 7 millions d'euros. Cette prise de participation d'une collectivité territoriale dans le capital d'une entreprise privée est courante pour les Länder voisins mais encore peu fréquente en France. Un des rares cas antérieurs est celui de l'entrée de la Région Poitou-Charentes dans le capital du petit constructeur automobile Heuliez ${ }^{11}$.

Dans cette recherche de capitaux, GECI Aviation, coté sur le marché Alternext de NYSE Euronext, a par ailleurs procédé, au début de l'été, à une ouverture de capital de 20 millions d'euros couverts à hauteur de $96 \%$.

L'aide de la Région est aussi matérielle. Elle est maître d'ouvrage de la réalisation de la zone d'activités entièrement consacrée à Sky Aircraftsur laquelle vont être construits progressivement entre 2010 et 2014 les différents bâtiments nécessaires à la production de l'avion (figure 5). L'aménageur est la SEBL (Société d'Equipement du Bassin Lorrain), une des plus vieilles sociétés d'équipement françaises et la société d'aménagement la plus importante de Lorraine.

Figure 5 : Maquette du futur site de Sky Aircraft

\section{Sky Aircraft : un site industriel de 22 hectares sur la base aérienne de Chambley-Bussières}
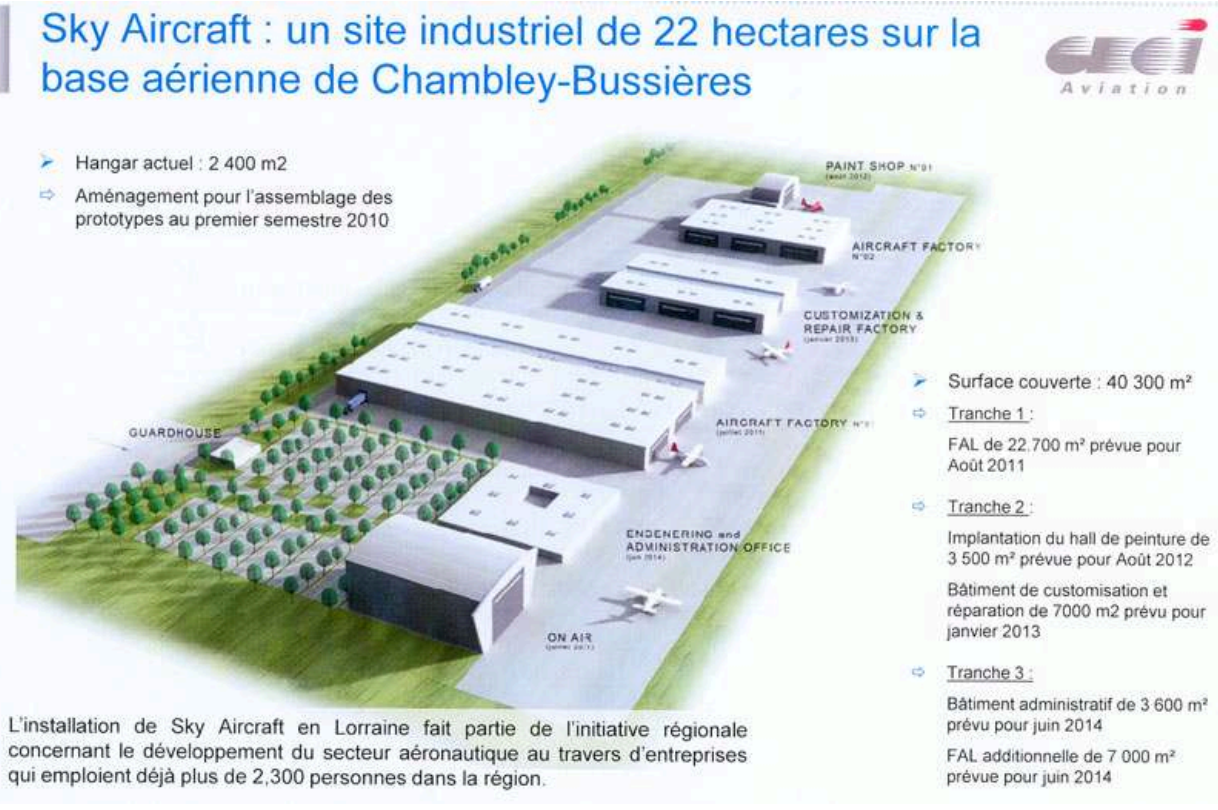

(c) GECI International

\section{B. Faire face à une crise de confiance}

Durant ces deux années, si le soutien de la majorité politique du conseil régional n'a pas failli, il est à noter que depuis le début du deuxième mandat du président, l'enthousiasme et la bienveillance dont a bénéficié l'entreprise tant dans la presse que parmi les élus lorrains ont été entamés d'une part par les réserves voire l'hostilité de certains élus mais aussi de membres du CES, et d'autre part par une série d'articles 
parus dans la presse régionale doutant ouvertement de la réalisation de l'avion « made in Lorraine $»^{12}$. Le principal argument développé par l'Est Républicain et repris dans d'autres articles durant tout le mois de juin 2010 est celui du non respect du lourd processus de certification auprès de l'EASA(Agence Européenne de la Sécurité Aérienne). Depuis, la procédure d'agrément a été ouverte. C'est une procédure longue et coûteuse s'étalant sur quelques dizaines de mois. S'il n'est pas possible d'apprécier s'il y a eu effectivement retard ou non dans cette démarche obligatoire, force est de constater, en revanche, qu'une baisse de $14 \%$ du cours de l'action GECI Aviation a été enregistrée le 28 juin, date du début de lever de fonds par GECI. L'entreprise devait espérer un climat plus favorable pour son extension de capital. Epiphénomène? Manœuvre politique de déstabilisation de l'entreprise, du programme Skylander ou du grand projet de la Région? Ou manifestation d'une crainte réitérée liée à d'anciennes désillusions causées par des « chasseurs de primes »?

\section{Retenir durablement une main d'œuvre extérieure à la région}

28 A l'écart des bureaux de presse, des états-majors, les équipes d'ingénieurs travaillent et «se concentrent sur l'essentiel» au calme dans cet espace rural de faible densité. Après l'étude de faisabilité, la deuxième phase du programme dénommée PDR (Preliminairy Design Review) c'est-à-dire celle des plans et de l'identification des fabricants potentiels est maintenant terminée; celle des dessins de fabrication est en cours. Ce travail de conception est assuré essentiellement par des ingénieurs de l'aéronautique recrutés par Sky Aircraft ou par des personnels de GECI International en déplacement à Chambley. Du fait de la spécificité du produit et de l'absence de tradition aéronautique en Lorraine, la quasi-totalité des ingénieurs travaillant à Chambley est extérieure à la région. Ils sont soit étrangers (plus de dix nationalités représentées), soit français originaires du Sud-Ouest ou de la région parisienne. Si la création d'emplois n'a encore que peu profité aux Lorrains, l'implantation de Sky Aircraft a-telle développé une économie résidentielle? Non, malgré l'installation de quelques salariés avec leurs familles dans les communes environnantes. En l'absence d'un parc locatif suffisant et attirant, une part importante du personnel réside à Metz, à 25-30 km de Chambley, en particulier à proximité de la gare ferroviaire ; chaque fin de semaine, une partie du personnel de Sky Aircraft s'échappe pour retrouver les familles restées dans des environnements jugés plus amènes et mieux équipés. Le recrutement de salariés hautement qualifiés et leur maintien sur le site de Chambley semblent constituer une préoccupation majeure, une réelle difficulté pour la direction de GECI qui ne parvient pas à freiner un turn over important. Au cours de l'été 2010, la direction a même étudié la possibilité de mettre en place un système de travail alternatif : trois semaines de travail à Chambley, une semaine de travail à Toulouse. Les principaux griefs évoqués pour justifier ou expliquer le refus des conjointes et des enfants des cadres de GECI de s'installer en Lorraine sont le déficit d'image de la région, la très grande ruralité de cette partie méridionale du Pays Haut constitué de très petits villages $^{13}$ qui manquent d'équipements d'éducation, de santé, de loisirs. De toute évidence, l' « envie de campagne » lorraine n'est pas partagée par tous.

Une implantation davantage périurbaine aurait-elle facilité les recrutements et une installation plus pérenne? Le recrutement de salariés nécessaires à la phase 
d'assemblage, d'essai des prototypes puis de production industrielle ancrera-t-elle davantage l'entreprise dans le territoire?

\section{Conclusion}

Cette recherche sur l'implantation de Sky Aircraft dans l'espace rural lorrain nous conduit à considérer cette localisation comme atypique ; en effet, cette jeune entreprise de haute technologie, qui n'a pas disjoint spatialement conception et production, semble avoir minimisé un des facteurs de localisation devenu de plus en plus important, notamment pour ce type d'industrie, un facteur immatériel, celui de la prise en compte des compétences et des qualifications. Cette contribution souligne aussi les relations étroites entre la sphère entrepreneuriale et les sphères politiques régionales et nationales, et sans aucun doute le poids du politique dans la décision finale de l'entreprise. GECI a été séduite par la réactivité de la Région; elle n'a probablement pas choisi Chambley mais commence à apprécier la présence du cluster Aériades. Une partie des fournisseurs de Sky Aircraft devrait être des entreprises implantées dans le Grand Est. La publication récente de la sélection des principaux équipementiers et fournisseurs (supply-chain), indique, pour l'instant, le choix de la société Leach $(\text { Sarralbe })^{14}$. La greffe de cette nouvelle entreprise de haute technologie, extérieure au territoire régional, générera-t-elle une dynamique de territoire innovant (Fache, 2002) ? Elle n'est encore qu'un objet géographique inachevé.

\section{BIBLIOGRAPHIE}

Bonnet J., Broggio C., 2010, Entreprises et territoires, Paris, Ellipses, 238 p.

Conseil Régional de Lorraine, 2006, Schéma régional de développement économique, 68 p., consulté le 21 décembre 2010, URL : http://www.lorraine.eu/jahia/jahia/cache/offonce/pid/18

Daviet S., 2004, «L'évolution du concept d'innovation : entrepreneurs, territoires et réseaux » in Innovation, Industrie et Recherche, Cahiers nantais, n62-63, p. 5-13

Fache J., 2002, Les territoires de la haute technologie. Eléments de géographie, Rennes, PUR, coll. Didact Géographie, $158 \mathrm{p}$.

Fischer A., 1999, «Quelques réflexions sur l'entreprise et le territoire » in Fischer A., Malézieux J., Industrie et aménagement, Paris, L'Harmattan, coll. Villes et entreprises, p.11-16.

Fischer A., 1999, « Les régions anciennement industrialisées et les effets géographiques des technologies nouvelles », in Fischer A., Malézieux J., Industrie et aménagement, Paris, L'Harmattan, coll. Villes et entreprises, p.49-64

GECI International, 2010, Rapport financier annuel exercice 2009-2010, mis en ligne le 06 août 2010, consulté le 22 décembre 2010, URL : http://www.geci.net/files/

FRPDF20100806_rapport_financier_annuel.pdf) 
Long X., 2004, «Innovation et gouvernance territoriale » in Innovation, Industrie et Recherche, Cahiers nantais, $\mathrm{n}^{\circ} 62-63$, p. 27- 32

Mérenne-Schoumaker B., 2002, La localisation des industries. Enjeux et industries, Rennes, PUR, coll. Didact Géographie, 243 p.

Renard-Grandmontagne C, 2004, Les zones d'activités : espaces pionniers à la périphérie des agglomérations. Le cas lorrain, Thèse Nouveau Régime, Université Nancy2, 458 p.

Renard-Grandmontagne C., 2010, « Les anciennes bases de l'OTANen Lorraine : quelles réutilisations ? », BAGF, $\mathrm{n}^{\circ}$ 3, p. 408-419

Sites consultés :

- www.aeriades.org, 21 décembre 2010

- www.business-in-lorraine.com, 21 décembre 2010

- www.datar.gouv.fr/fr_1/actualites_50/bref_57/archives_bref_58/actualise_sa_2355.html, dernière mise à jour le 29/07/2009, consulté le 07/09/2010.

\section{NOTES}

1. Le Républicain Lorrain, 6 septembre 2008

2. "Aéronautique, l'envol d'une filière lorraine?", Le journal des entreprises, 3/10/ 2008; "Serge Bitboul, avionneur en mal de confiance », Le Républicain Lorrain, 1/07/2010

3. Plaquette de la Région Lorraine, Point d'étape du Comité de pilotage, jeudi 17 décembre 2009

4. Effectif au 31 mars 2010, Rapport financier annuel exercice 2009-2010, www.geci.net onglet investisseurs.

5. Les deux seules implantations extra-européennes de GECI International sont Johannesburg et Shanghai.

6. La liste des membres du cluster Aériades est consultable sur le site www.aeriades.org

7. Les établissements déjà implantés bénéficient d'une АОт (autorisation d'occupation temporaire): entreprise de menuiserie sous traitante de Lapeyre (320 salariés), société de formation à la sécurité routière.

8. Le classement en zone AFR autorise l'attribution de subvention à hauteur de $15 \%$ du projet global. Le zonage AFR pour Chambley et les communes voisines a été confirmé en 2009. La carte AFR détaillée France entière 2007-13 est consultable sur le site de la Datar: http:// www.datar.gouv.fr/fr_1/actualites_50/bref_57/archives_bref_58/actualise_sa_2355.html

9. "L'heure est à la compensation " in "Aéronautique, l'envol d'une filière lorraine? ", Le Journal des entreprises, 3/10/ 2008

10. L'Est Républicain, 2 septembre 2008

11. La Région Poitou-Charentes a décidé d'entrer dans le capital d'Heuliez Véhicule Electrique à hauteur de 5 millions d'euros, soit 31\% du capital, en mai 2009.

12. Les premiers articles ont été rédigés par Marcel Gay et publiés dans l'Est Républicain du 7 juin 2010

13. Au recensement 2006, Chambley-Bussières (445 hab), Hagéville (112 hab), Dampvitoux (72 hab), Puxieux (240 hab), Dommartin-La-Chaussée (51 hab), St Julien-les-Gorze (147 hab), Charey (73 hab).

14. La société Leach International, filiale du groupe américain Esterline, est spécialisée dans les composants électroniques principalement pour l'aéronautique civile et militaire $(70 \%$ de 
l'activité). Elle est un des premiers membres du cluster Aeriades. Elle doit fournir le circuit électrique primaire du Skylander.

\section{RÉSUMÉS}

Plus de dix ans après l'inauguration de l'usine de la Smart, près de Sarreguemines, l'implantation du nouvel avionneur Sky Aircraft, constitue le dernier grand projet internationalement mobile en Lorraine. La filiale de GECI International, entreprise innovante, a finalement choisi une région française en quête de reconversion et d'attractivité, comme terre d'élection. Cette implantation est le fruit d'intérêts partagés par l'entreprise etla Région Lorraine : la recherche de partenaires publics prêts à accompagner un projet industriel d'une part, celle du développement d'une filière de haute technologie d'autre part. La greffe de cette nouvelle activité, sur l'ancienne base aérienne de l'OTAN, à Chambley, n'est cependant pas encore pleinement réalisée.

More than ten years after the unveiling of the plant of Smart, near Sarreguemines, the establishment of the new aircraft manufacturer Sky Aircraft, constitutes the last big plan internationally mobile in Lorraine. The subsidiary of GECI International, innovative firm, finally chose a French region in search of restructuring and of attractiveness, as earth of election. This establishment is the fruit of interests shared between the firm and the Lorraine Region: the research of public partners ready to accompany an industrial plan on one hand, that of the development of a course of study of high-tech on the other hand. The grafting of this new activity, on the ancient air base of NATO, in Chambley, is not however accomplished still entirely.

Mehr als zehn Jahre nach der Eröffnung des Smart-Werkes in der Nähe von Saargemünd bildet die Ansiedlung des neuen Flugzeugbauers Sky Aircraft das letzte international Großprojekt in Lothringen. Die Tochergesellschaft von GECI International, ein innovatives Unternehmen hat sich für eine französische Gegend auf der Suche nach Umwandlung und Attraktivität als Wahlheimat entschlossen. Diese Ansiedlung ist das Produkt gemeinsamer Interesse des Unternehmens und der Gegend Lothringen: einerseits die Suche nach öffentlichen Partner, die bereits sind, ein industrielles Projekt zu unterstützen und andererseits die Entwicklung einer High-tech Branche. Die Transplantation dieser neuen Aktivität auf dem ehemaligen OTAN Luftwaffenstützpunkt bei Chambley ist jedoch noch nicht gelungen.

\section{INDEX}

Schlüsselwörter : Ansiedlungsstrategie, Flugzeugbau, Innovation, politischer Akteur, Unternehmen

Keywords : aeronautics, firm, innovation, political actor, setting up strategy

Mots-clés : acteur politique, aéronautique, entreprise, innovation, stratégie d'implantation 
AUTEUR

\section{COLETTE RENARD-GRANDMONTAGNE}

MCF Université Nancy 2, CERPA - 3 place Godefroy de Bouillon - 54015 Nancy cedex -

colette.renard-grandmontagne@univ-nancy2.fr 